\title{
PINK1-Interacting Proteins: Proteomic Analysis of Overexpressed PINK1
}

\author{
Aleksandar Rakovic, Anne Grünewald, Lisa Voges, Sarah Hofmann, Slobodanka Orolicki, \\ Katja Lohmann, and Christine Klein
}

\begin{abstract}
Section of Clinical and Molecular Neurogenetics, Department of Neurology, University of Lübeck, Maria-Goeppert-Straße 1, 23562 Lübeck, Germany
\end{abstract}

Correspondence should be addressed to Christine Klein, christine.klein@neuro.uni-luebeck.de

Received 22 November 2010; Accepted 22 December 2010

Academic Editor: Charleen T. Chu

Copyright ( $\odot 2011$ Aleksandar Rakovic et al. This is an open access article distributed under the Creative Commons Attribution License, which permits unrestricted use, distribution, and reproduction in any medium, provided the original work is properly cited.

Recent publications suggest that the Parkinson's disease- (PD-) related PINK1/Parkin pathway promotes elimination of dysfunctional mitochondria by autophagy. We used tandem affinity purification (TAP), SDS-PAGE, and mass spectrometry as a first step towards identification of possible substrates for PINK1. The cellular abundance of selected identified interactors was investigated by Western blotting. Furthermore, one candidate gene was sequenced in 46 patients with atypical PD. In addition to two known binding partners (HSP90, CDC37), 12 proteins were identified using the TAP assay; four of which are mitochondrially localized (GRP75, HSP60, LRPPRC, and TUFM). Western blot analysis showed no differences in cellular abundance of these proteins comparing PINK1 mutant and control fibroblasts. When sequencing LRPPRC, four exonic synonymous changes and 20 polymorphisms in noncoding regions were detected. Our study provides a list of putative PINK1 binding partners, confirming previously described interactions, but also introducing novel mitochondrial proteins as potential components of the PINK1/Parkin mitophagy pathway.

\section{Introduction}

Parkinson's disease (PD) is a progressive neurodegenerative disorder with three cardinal manifestations: tremor, rigidity, and bradykinesia. In about $25 \%$ of all PD patients, at least one additional affected family member can be found, likely pointing to a direct, genetic cause of the disease. To date, eight genes have been confirmed to be associated with $\mathrm{PD}$ [1].

Besides the mutational analysis of PD-linked genes, the search for potential interactions between the protein products of these genes has recently gained increasing importance. Since the identification of the second monogenetic PD gene product Parkin, a common pathway leading to dopaminergic neurodegeneration has been proposed. To date, several studies reported such connections.

First, Shimura et al. hypothesized that Parkin plays a role in the coregulation of Alpha-synuclein (SNCA). The group identified a protein complex in normal human brain that included the E3 ubiquitin ligase Parkin, UBCH7 as its associated E2 ubiquitin-conjugating enzyme, and a novel form of SNCA as its substrate [2]. Later, an early-onset PD patient with a heterozygous missense mutation in both the $D J-1$ and the PTEN-induced putative kinase 1 (PINK1) gene has been described. Additionally, overexpression of DJ-1 and PINK1 in SHSY-5Y cells revealed that the wildtype as well as the mutant forms of both proteins interact, and DJ-1 stabilized PINK1 [3]. Furthermore, Drosophila pink1 and parkin lossof-function mutants showed a similar mitochondrial phenotype. Since the pinkl-related abnormalities could be rescued by parkin overexpression but not vice versa, it was suggested that pink1 acts upstream of parkin in a common pathway [46]. Recent studies provided evidence that the PINK1/Parkin pathway promotes mitochondrial fission as an initial step of mitophagy by ubiquitination of Mitofusins [7-9].

Still, only the minority of hereditary forms of PD can be explained by a mutation in one of the nine PD-associated 
genes [10]. Therefore, this research focuses not only on proteins known to be involved in PD but also on novel interactors.

In the present study, we employed tandem affinity purification (TAP) to isolate proteins that are directly associated with PINK1. Using this approach, we aimed at a better characterization of the PINK1/Parkin mitophagy pathway.

\section{Material and Methods}

2.1. Patients. All patients underwent a standardized neurological examination performed by a movement disorders specialist. For sequencing of one of the candidate genes, Leucine-rich PPR motif-containing (LRPPRC), 46 patients with atypical Parkinsonism including features of dementia, depression, or rapid disease progression were included in the present study. After obtaining informed consent, we collected blood from all patients for DNA extraction according to a published protocol [11].

Furthermore, fibroblasts from three PD patients with a homozygous p.Q456X PINK1 mutation and from two agematched mutation-negative healthy controls were included in the study. Clinical features of these mutation carriers are described elsewhere $[12,13]$.

2.2. Tissue Culture. HEK cells and fibroblasts were cultured in Dulbecco's modified Eagle's medium (PAA Laboratories) and supplemented with $10 \%$ fetal bovine serum and $1 \%$ penicillin-streptomycin. All cells were maintained at $37^{\circ} \mathrm{C}$ in a saturated humidity atmosphere containing $5 \% \mathrm{CO}_{2}$. Passage numbers $<10$ were used for all experiments. To inhibit the mitochondrial membrane potential, fibroblasts were treated with the potassium ionophore valinomycin (1 $\mu \mathrm{M}$, Sigma).

\subsection{Protein Isolation by TAP and Mass Spectrometry (MS).} The InterPlay Mammalian TAP System was employed according to the manufacturer's (Stratagene) protocol. In brief, human control RNA was extracted using the RNeasy Mini Kit (Qiagen) and PINK1 cDNA was synthesized using Superscript II Reverse Transcriptase in combination with oligo dT primers (Invitrogen). Full-length PINK1 cDNA was cloned into the PCTAP expression vector, which encodes two tandem affinity tags (a streptavidin binding peptide and a calmodulin binding peptide) after its multiple cloning site. Next, $10^{8}$ HEK cells were transiently transfected with the pCTAP-PINK1 vector by means of the $\mathrm{Ca}^{2+} \mathrm{PO}_{4}$ method [14]. After $24 \mathrm{~h}$, cells were harvested and resuspended in lysis buffer supplemented with protease inhibitors (Sigma). Cells underwent three rounds of freeze-thawing, cell debris was pelleted by centrifugation at $16,000 \times \mathrm{g}$ for $10 \mathrm{~min}$, and the supernatant collected. Next, $2 \mathrm{mM}$ EDTA, $10 \mathrm{mM} \beta$ mercaptoethanol, and the InterPlay streptavidin resin were added to the cell lysate and incubated at $4^{\circ} \mathrm{C}$ while rotating for $2 \mathrm{~h}$. The resin was collected by centrifugation at $1,500 \times \mathrm{g}$ for $5 \mathrm{~min}$, washed twice, and incubated in biotin-containing buffer for $30 \mathrm{~min}$ at $4^{\circ} \mathrm{C}$ to elute the bound protein complexes. To further purify the protein complexes, calmodulin resin and a calcium-containing buffer were added to the supernatant. After $2 \mathrm{~h}$ of incubation at $4^{\circ} \mathrm{C}$, the resin was collected (centrifugation at $1,500 \times \mathrm{g}$ for $5 \mathrm{~min}$ ) and washed twice. From the calmodulin resin, protein complexes were eluted by adding EDTA-containing buffer and incubation for $30 \mathrm{~min}$ at $4^{\circ} \mathrm{C}$ on a rotator. The resulting eluate was concentrated by means of trichloroacetic precipitation and subsequently resuspended in radioimmunoprecipitation assay (RIPA) buffer (50 mM Tris- $\mathrm{HCl}$ pH7.6, $150 \mathrm{mM} \mathrm{NaCl}$, $1 \%$ DOC, and $1 \%$ NP-40) with protease and phosphatase inhibitors (Roche Diagnostics). Next, purified proteins were resolved by one-dimensional SDS-polyacrylamide gel electrophoresis (SDS-PAGE) and visualized on the gel by silver staining as published [15]. In a control experiment, the pulldown approach was performed with beads only. Bands which appeared on the gel from the PINK1 overexpression experiment but not the control experiment were excised and sent for MS analysis to the Taplin Biological Mass Spectrometry Facility, Harvard Medical School, Boston, USA. According to the quality requirements of the facility, a protein can only be considered as interactor if two or more peptides match the respective protein in the Swiss-Prot database.

2.4. Knock-down Approach. For PINK1 and LRPPRC knockdown, Hs_PINK1_4_HP (Qiagen) and LRPPRC UTR (complementary to the segment of the $3^{\prime}$ UTR region of LRPPRC [16]) siRNAs were used with a final concentration of $50 \mathrm{nM}$. Scrambled siRNA (Silencer negative control 1 siRNA [Ambion]) with no known mammalian homology served as negative control (final concentration $50 \mathrm{nM}$ ). For transfection, the Nucleofector Device (Lonza) was used.

2.5. Mitochondrial Preparation. Mitochondria were isolated from fibroblasts as previously described [17]. In brief, cells were harvested and homogenized in buffer containing $250 \mathrm{mM}$ sucrose, $10 \mathrm{mM}$ Tris, and $1 \mathrm{mM}$ EDTA, pH7.4. After that, nuclei and unbroken cells were removed by centrifugation at $1,500 \times \mathrm{g}$ for $20 \mathrm{~min}$. The supernatant containing intact mitochondria was transferred into a new tube and centrifuged at $12,000 \times \mathrm{g}$ for $10 \mathrm{~min}$. The resulting supernatant (cytosolic fraction) was transferred into another new tube and the mitochondria-enriched pellet (mitochondrial fraction) was dissolved in RIPA buffer containing protease and phosphatase inhibitors (Roche Diagnostics). Cytoplasmic fractions were concentrated by using Centricon YM-10 devices (Millipore) according to the manufacturer's instructions.

2.6. Protein Extraction. Proteins were extracted using RIPA buffer containing $0.1 \%$ SDS. Cells or mitochondria-enriched pellets were dissolved in the appropriate amount of buffer and incubated on ice for $30 \mathrm{~min}$. After that, the lysates were centrifuged at $16,000 \times \mathrm{g}$ for $20 \mathrm{~min}$ at $4^{\circ} \mathrm{C}$. The supernatant was transferred into a new tube for further processing.

2.7. Western Blot Analysis. SDS-PAGE was performed using NuPAGE 4\%-12\% Bis-Tris gels (Invitrogen). After electrophoresis, proteins were transferred to the nitrocellulose 


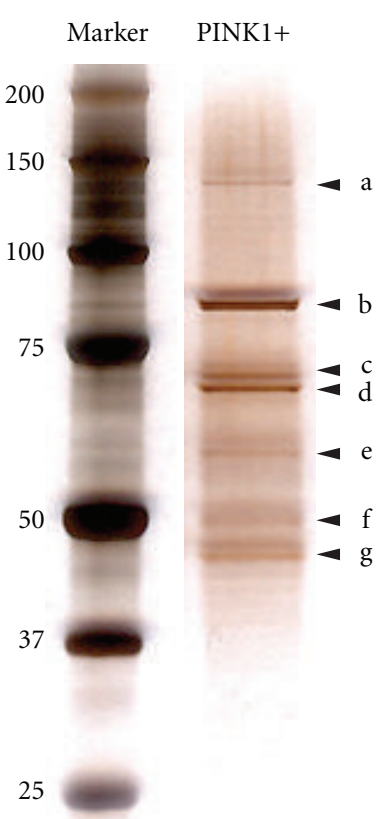

(a)

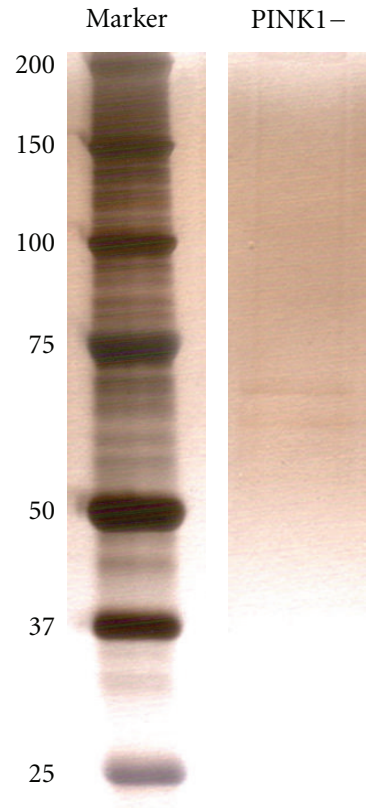

(b)

FIGURE 1: SDS-PAGE result after TAP with overexpressed PINK1. (a) A TAP approach was performed with HEK cells overexpressing PINK1 $(\mathrm{PINK} 1+)$. Purified proteins were resolved by SDS-PAGE and visualized on the gel by silver staining. Protein bands which were excised from the gel are marked by an arrow head (a-g). (b) In a control experiment, the pull-down approach was performed with beads only (PINK1-). A molecular weight marker was used to estimate the size of the detected protein bands.

membrane (Protran) and probed with antibodies raised against $\beta$-actin (Sigma), Mortalin (GRP75, Abcam), Heat shock $60 \mathrm{kDa}$ protein (HSP60, Cell Signalling), Mitochondrially encoded cytochrome c oxidase I (MT-CO1, MitoSciences), Voltage-dependent anion channel 1 (VDAC1, Abcam) and Elongation factor TU (TUFM, Abcam). An antibody against LRPPRC was kindly provided by Professor S. Piñol-Roma, Brookdale Department of Molecular, Cell and Developmental Biology, Mount Sinai School of Medicine, New York, USA [18].

2.8. Mutational Screening. All 38 coding LRPPRC exons and flanking intronic regions were sequenced on an $\mathrm{ABI}$ 3100 Genetic Analyzer. Primers and PCR conditions are summarized in Supplementary Table 1 available online at doi:10.4061/2011/153979.

\section{Results and Discussion}

3.1. New PINK1 Interactors. Using a TAP approach with overexpressed PINK1 (PINK1+), or empty beads (PINK1-) we identified interactors of the target protein. The resulting TAP eluates were resolved by SDS-PAGE. Silver staining was used to visualize protein bands on the PAGE gels. Seven bands were excised from the PINK1+ gel (Figure 1(a), a-g) which were not detectable on the PINK1- gel (Figure 1(b)) and analyzed by MS (Table 1).

A total of 14 proteins were identified which met the requirement of having two or more peptides matched to it by the database-searching program. Out of those, seven are predominantly found in the cytoplasm (Heat shock $90 \mathrm{kDa}$ proteins alpha and beta, Heat shock $70 \mathrm{kDa}$ proteins 1, 2, 8, and 1-like, and Hsp90 cochaperone Cdc37), two are components of microtubuli (Tubulin alpha-1C chain and Tubulin alpha-3C/D chain), one is associated with the endoplasmic reticulum ( $78 \mathrm{kDa}$ Glucose-regulated protein) and four are mitochondrially localized (GRP75, HSP60, LRPPRC, and TUFM) (for details on subcellular localization see: http://expasy.org/sprot/). Of note, also PINK1 itself was identified by MS in most of the analyzed bands on the PAGE gel (Figure 1(a), b-g).

To our knowledge, only the interaction between the HSP90/CDC37 chaperone system and PINK1 has been described so far $[19,20]$. CDC37 is a molecular cochaperone that functions with HSP90 to promote folding of kinases [21]. With respect to PINK1, the chaperone system was found to influence the protein's subcellular distribution. The authors of this study proposed that the HSP90/CDC37/PINK1 complex is destined for a translocation that leads to PINK1 processing, whereas in the absence of HSP90 in the complex, PINK1 might be attached to mitochondria as full-length precursor [19].

Given that PINK1 was reported to be associated with mitochondria $[17,22]$, we focused primarily on the mitochondrial proteins GRP75, HSP60, LRPPRC, and TUFM in the ensuing experiments.

3.2. Cellular Abundance of GRP75, HSP60, LRPPRC, and TUFM. First, we determined the quality of the noncommercially available LRPPRC antibody by means of a knock-down 


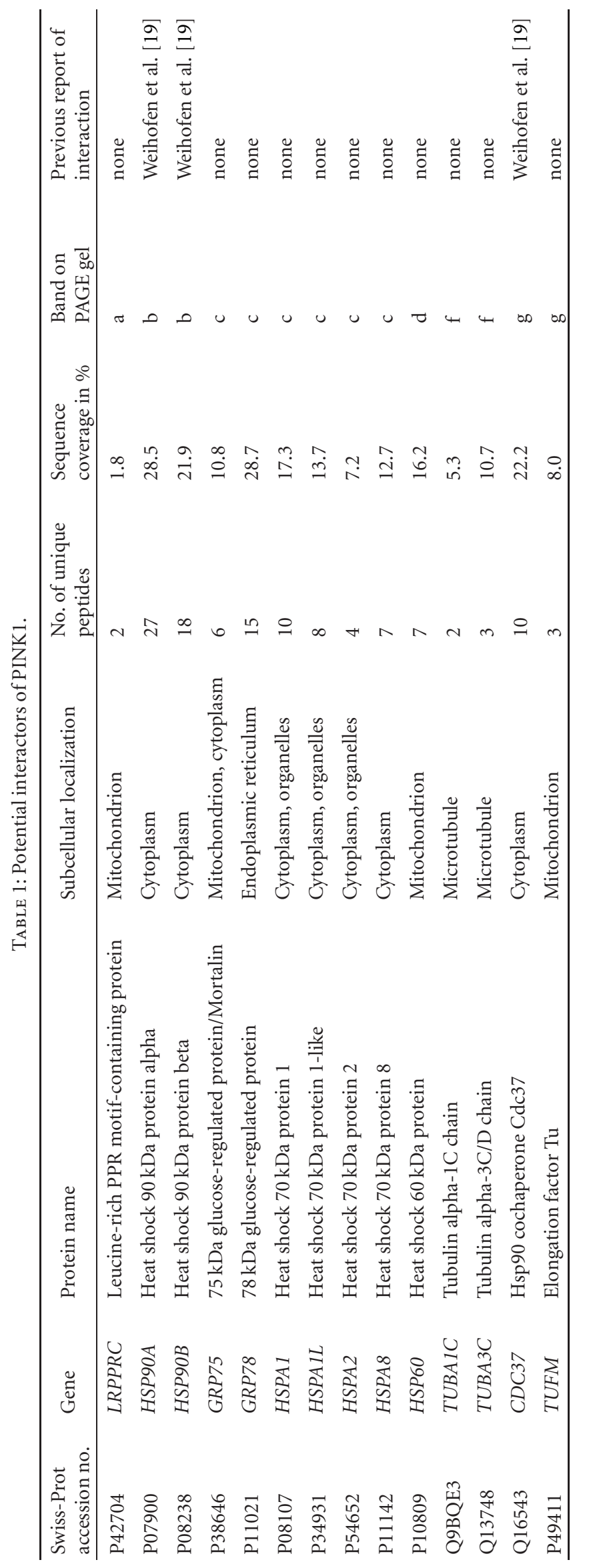




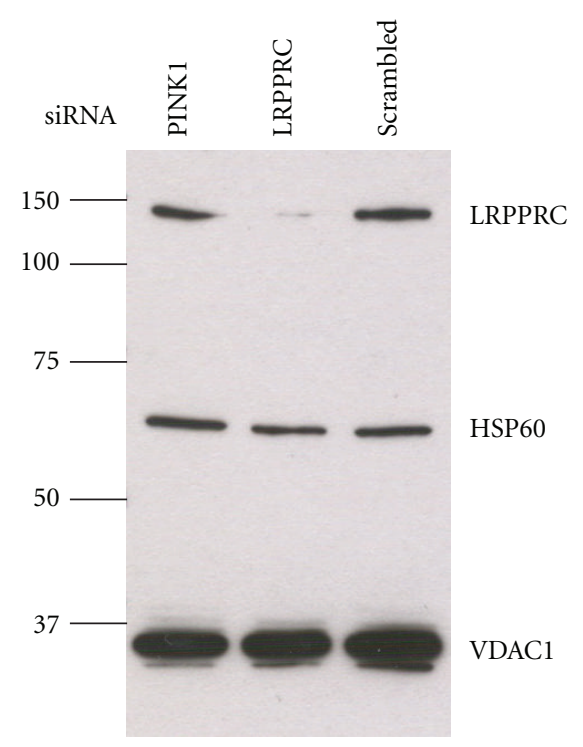

FIGURE 2: Specificity of an anti-LRPPRC antibody. Fibroblasts were incubated with PINK1 siRNA, LRPPRC siRNA, or scrambled siRNA for $24 \mathrm{~h}$. Whole cell lysates were analyzed by Western blotting with an antibody against LRPPRC. LRPPRC levels decreased only when LRPPRC siRNA was employed, confirming the specificity of the anti-LRPPRC antibody used in our study. The mitochondrial markers HSP60 and VDAC1 served as loading controls. HSP60: Heat shock $60 \mathrm{kDa}$ protein; LRPPRC: Leucine-rich PPR motifcontaining protein; VDAC1: Voltage-dependent anion channel 1.

approach. This experiment showed a drop in LRPPRC levels when siRNA against $L R P P R C$ was employed but not when scrambled siRNA was used, confirming the specificity of the antibody. LRPPRC protein levels were also not affected by a PINK1 knockdown (Figure 2).

Next, the abundance of GRP75, HSP60, LRPPRC, and TUFM was investigated in mitochondrial fractions from control and PINK1-mutant fibroblasts. This experiment revealed comparable levels of GRP75, HSP60, and LRPPRC in both groups (Figures $3(\mathrm{a})$ and 3 (b)-left half). The abundance of TUFM was variable in all investigated samples showing no clear trend when comparing mutants and controls (Figure 3(a)).

Furthermore, we tested the quality of the GRP75 antibody by investigating GRP75 in the cytosolic fraction under valinomycin stress conditions. GRP75 is a mitochondrial matrix chaperone, synthesized as a 679 -amino acid preprotein, which contains a 51-residue $\mathrm{N}$-terminal mitochondrial targeting sequence (MTS). After the membrane potentialdependent import into mitochondria, it is cleaved into the mature protein which is $\sim 5.5 \mathrm{kDa}$ shorter than the preprotein [23-25]. When treating cells with the mitochondrial membrane potential inhibitor valinomycin, the full-length form of GRP75 (MTS-GRP75) was detected in the cytosolic fractions from mutants and controls. Consequently, we considered the anti-GRP75 antibody as specific. In both groups, the levels of MTS-GRP75 were comparable (Figure 3(b)right half). The abundance of the processed form of GRP75 in the cytosolic fraction was likely due to contamination with the mitochondrial fraction, although previous studies have shown that GRP75 can also be cytosolically localized [26].

Though our Western blot results are not supporting a direct link between PINK1 and any of the detected mitochondrial proteins, it should be noted that GRP75, HSP60, and LRPPRC have been identified by proteomic analysis as potential interactors of Parkin earlier [27]. Furthermore, their molecular functions render them interesting targets in the context of PD.

GRP75 serves as a major mitochondrial molecular chaperone and plays a key role in the import and partitioning of nuclear-encoded proteins within the two mitochondrial membranes and the matrix [28-30]. Furthermore, GRP75 seems to function in the management of oxidative stress via the PD-associated protein DJ-1. Mutations in DJ-1 were found to weaken the protein's interaction with GRP75 [31, 32]. GRP75 has also been described as an antiapoptotic agent. By binding of the transcription regulator p53, GRP75 prevents the formation of the proapoptotic $\mathrm{p} 53 / \mathrm{Bcl}-\mathrm{xL} / \mathrm{Bcl}-2$ complex $[33,34]$. Furthermore, putative mutations in GRP75 were suggested to contribute to the risk of developing PD [35] and a decrease in GRP75 expression was detected in PD patient brains compared to controls [36].

HSP60 is a mitochondrial chaperone responsible for the transport of nuclear-encoded proteins via the mitochondrial membranes and their refolding in the matrix $[37,38]$ and has been linked to the pathogenesis of Alzheimer's disease. Apparently, HSP60 provides protection against intracellular $\beta$-amyloid stress through maintenance of mitochondrial respiratory complex IV activity [39]. Complex IV deficiency in turn, has been implicated in PD $[40,41]$ opening the possibility for a role of HSP60 in the pathogenesis of the disease.

LRPPRC has been linked to cytochrome $\mathrm{C}$ oxidase deficiency. Mutations in the gene lower MT-CO1 and MTCO3 mRNA levels and, in turn, impair complex IV assembly $[42,43]$. Recent functional studies further strengthen the link between LRPPRC and mitochondrial RNA metabolism [44, $45]$. However, when we compared MT-CO1 protein levels in fibroblasts from PINK1 mutants and controls, no differences were observed (Figure 3(a)). Furthermore, LRPPRC was identified as a component of the PGC- $1 \alpha$ complex which itself is also linked to energy homeostasis in the cell [46]. Like in the case of HSP60, LRPPRC's impact on the respiratory chain offers a potential connection with PD.

TUFM is part of the translational apparatus of mitochondria. During protein biosynthesis, it mediates the GTPdependent binding of aminoacyl-tRNA to the A-site of ribosomes [47]. Additional described functions of TUFM comprise recognition and translocation of cotranslationally damaged proteins to the proteasome [48], rearrangement of cytoskeletal components $[49,50]$, and regulation of cell survival [51]. Mutations in the TUFM gene cause combined oxidative phosphorylation deficiency type 4 due to decreased mitochondrial protein synthesis [52]. Interestingly, however, there is also a report connecting TUFM and PD, where TUFM was found to co-immunoprecipitate with Leucin-rich repeat kinase 2 which is encoded by the PARK8 gene LRRK2. Coincubation with recombinant TUFM reduced the kinase 


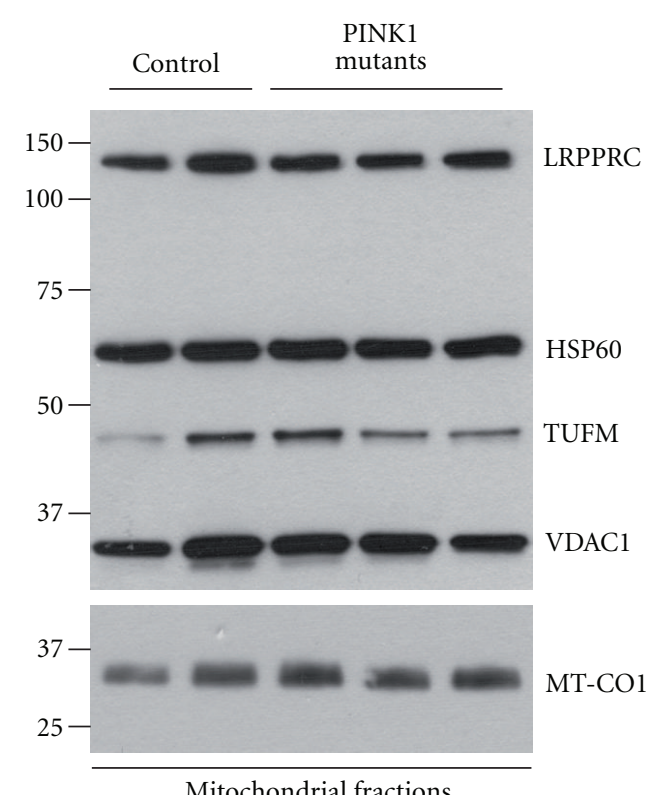

(a)
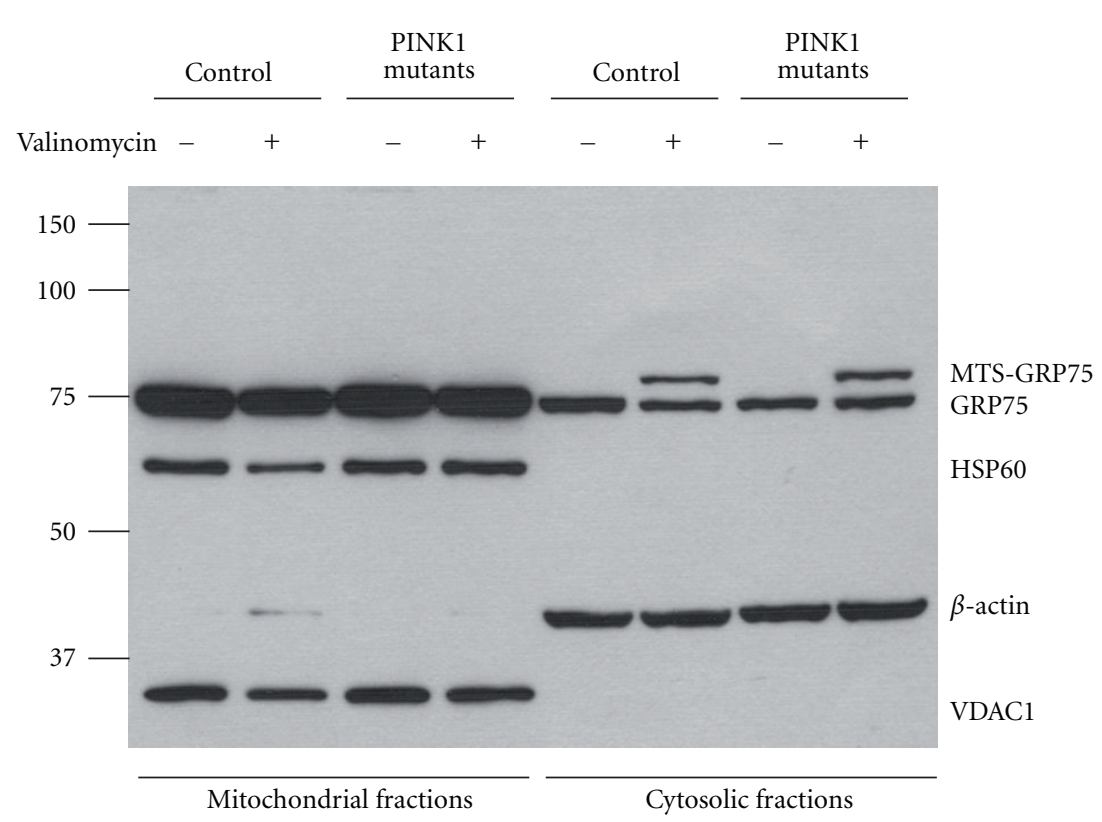

(b)

FIGURE 3: Cellular abundance of potential mitochondrial PINK1-interacting proteins. Mitochondrial and cytosolic fractions from fibroblasts were analyzed by Western blotting using antibodies against HSP60, LRPPRC, TUFM, MT-CO1, and GRP75. (a) The mitochondrial localization of LRPPRC and TUFM was confirmed, and no differences in their cellular abundance were detected when comparing PINK1 mutants and controls. Furthermore, the level of LRPPRC-associated MT-CO1 was not altered in PINK1 mutants. (b) In the mitochondrial fractions, the abundance of (processed) GRP75 was comparable in PINK1 mutants and controls under basal and valinomycin stress conditions $(1 \mu \mathrm{M}$ for $24 \mathrm{~h}$ ). In the cytosol, an additional band representative of accumulation of nonprocessed MTS-GRP75 was detected when cells were treated with the mitochondrial membrane inhibitor valinomycin. Due to a possible contamination of the cytosolic fraction with mitochondria and/or partially cytosolic localization of GRP75, also the processed form of the protein is apparent in this fraction. The mitochondrial marker VDAC1 and the cytosolic marker $\beta$-actin served as loading controls. GRP75: $75 \mathrm{kDa}$ glucose-regulated protein; HSP60: Heat shock $60 \mathrm{kDa}$ protein; LRPPRC: Leucine-rich PPR motif-containing protein; MT-CO1: Mitochondrially encoded cytochrome c oxidase I; MTS-GRP75: GRP75 with mitochondrial targeting sequence; TUFM: Elongation factor Tu; VDAC1: Voltage-dependent anion channel 1. 
TABLE 2: Allelic frequencies of sequence variations identified in LRPPRC.

\begin{tabular}{|c|c|c|c|c|c|}
\hline Gene position & DNA variation & NCBI no. & AF PD & AF DB & Database* \\
\hline $5^{\prime} \mathrm{UTR}$ & c. $-45 \mathrm{G}>\mathrm{A}$ & rs11124961 & $7.6 \%$ & $1.4 \%$ & pilot.1.CEU \\
\hline Exon 2 & c.246G>A (p.Q82Q) & rs6741066 & $66.7 \%$ & $65.5 \%$ & HapMap-CEU \\
\hline Intron 3 & IVS3-132C $>\mathrm{G}$ & rs6721144 & $6.8 \%$ & $13.3 \%$ & HapMap-CEU \\
\hline Intron 6 & IVS6-70T $>C$ & rs17031786 & $14.4 \%$ & $13.8 \%$ & HapMap-CEU \\
\hline Exon 9 & c.1068A>G (p.Q356Q) & rs4953042 & $16.3 \%$ & $19.2 \%$ & HapMap-CEU \\
\hline Intron 9 & IVS9+30A $>\mathrm{G}$ & rs7593842 & $15.2 \%$ & $12.7 \%$ & HapMap-CEU \\
\hline Intron 13 & IVS13+28T $>C$ & rs62135104 & $9.5 \%$ & $1.5 \%$ & pilot.1.CEU \\
\hline Intron 15 & IVS15+11C>G & rs58811869 & $7.8 \%$ & $13.9 \%$ & pilot.1.CEU \\
\hline Intron 17 & IVS17-28T >G & rs72877186 & $15.2 \%$ & $15.3 \%$ & pilot.1.CEU \\
\hline Intron 20 & IVS20-40A $>C$ & rs7594526 & $42.4 \%$ & $47.5 \%$ & HapMap-CEU \\
\hline Intron 22 & IVS $22+27 \mathrm{~T}>\mathrm{G}$ & rs28394191 & $43.5 \%$ & $40.3 \%$ & pilot.1.CEU \\
\hline Exon 23 & c. $2481 \mathrm{~A}>\mathrm{G}($ p.P827P) & rs115993634 & $1.1 \%$ & none & none \\
\hline Intron 27 & IVS27+26C $>\mathrm{T}$ & rs4952694 & $51.1 \%$ & $53.0 \%$ & AoD_Caucasian \\
\hline Intron 27 & IVS27-38A $>\mathrm{G}$ & none & $2.2 \%$ & none & none \\
\hline Intron 28 & IVS28+21C $>\mathrm{A}$ & rs7568481 & $43.5 \%$ & $47.4 \%$ & HapMap-CEU \\
\hline Intron 30 & IVS30+97T $>C$ & rs17424482 & $8.7 \%$ & $3.7 \%$ & HapMap-CEU \\
\hline Intron 32 & IVS32-3C $>\mathrm{T}$ & rs35113761 & $6.5 \%$ & none & none \\
\hline Intron 35 & IVS35+14C $>\mathrm{T}$ & rs3795859 & $15.2 \%$ & $15.0 \%$ & HapMap-CEU \\
\hline Intron 35 & IVS35+15C $>\mathrm{T}$ & rs76850904 & $8.7 \%$ & none & none \\
\hline Intron 36 & IVS36-42G $>C$ & none & $1.1 \%$ & none & none \\
\hline Exon 37 & c.4023T >C (p.Y1341Y) & none & $1.1 \%$ & none & none \\
\hline Intron 37 & IVS37+37G $>A$ & rs2955280 & $51.1 \%$ & $53.4 \%$ & HapMap-CEU \\
\hline $3^{\prime} \mathrm{UTR}$ & $* 399 \mathrm{G}>\mathrm{A}$ & none & $2.3 \%$ & none & none \\
\hline $3^{\prime} \mathrm{UTR}$ & $* 556 \mathrm{~A}>\mathrm{T}$ & rs1136998 & $7.6 \%$ & $8.3 \%$ & HapMap-CEU \\
\hline
\end{tabular}

Note: AF: allelic frequency, DB: database, PD: Parkinson's disease and * Only studies based on European populations included.

activity of LRRK2, whereas the GTPase activity remained unchanged [53].

3.3. LRPPRC Mutational Screen. Among the identified PINK1 interactors, LRPPRC is the only protein which is unequivocally linked to a neurodegenerative disorder. Mutations in LRPPRC are the cause of the French-Canadian type of Leigh syndrome (LSFC). LSFC patients suffer from progressive focal necrotizing lesions of the brainstem, basal ganglia, and cerebellum, accompanied by capillary proliferation. Besides metabolic acidosis, clinical features include generalized developmental delay, cerebellar signs, and a striking paucity of facial and limb movement, as well as hypomimia [46]. Given the presence of Parkinsonian signs in LSFC patients, we decided to sequence the 38 exons and flanking intronic regions of $L R P P R C$ in 46 patients with atypical PD with early onset and/or rapid disease progression and dementia. This mutational screen revealed 24 substitutions; four of which have not yet been reported in any database (Table 2). Four synonymous variations were detected in the coding region (c.246G $>A$ [p.Q82Q], c.1068A $>$ G [p.Q356Q], c.2481A>G [p.P827P], c.4023T>C [p.Y1341Y]). Seventeen changes were found in introns, one in the $5^{\prime}$ UTR and two in the $3^{\prime}$ UTR. The frequencies of most substitutions in our sample were similar to those reported in the NCBI SNP database (http://www.ncbi.nlm.nih.gov/) for studies based on populations of European origin, such as pilot.1.CEU, HapMap-CEU, and AoD_Caucasian. Interestingly, frequencies of SNPs c. $-45 \mathrm{G}>\mathrm{A}$, IVS13+28T $>\mathrm{C}$ and IVS30+97T $>$ C, were markedly higher than those reported in the databases. The significance of this finding needs to be investigated in a larger sample. The screening techniques used here allow, however, only for the identification of qualitative sequence changes. Therefore, although no single nucleotide changes of likely pathogenic relevance have been found in the LRPPRC gene, gene dosage variations cannot be excluded.

\section{Conclusions}

In the current study, TAP technology was employed for the first time to identify PINK1-associated proteins. These experiments resulted in a list of 14 putative PINK1 binding partners, confirming two reported interactions (HSP90 and CDC37), but also introducing four novel mitochondrially localized proteins (GRP75, HSP60, LRPPRC, or TUFM) as potential components of the PINK1/Parkin mitophagy pathway. Although preliminary results from protein expression and DNA sequencing analyses do not strengthen a link between the PINK1/Parkin pathway and any of these interactors, it cannot be excluded that their connection 
with the pathway may be more complex. Additional protein function studies, for instance under mitochondrial stress conditions, will be needed to fully characterize this potential link. In addition, future perspectives include association studies with SNPs in all identified genes in a larger PD patient sample.

\section{Acknowledgments}

The authors thank Professor S. Piñol-Roma, Brookdale Department of Molecular, Cell and Developmental Biology, Mount Sinai School of Medicine, New York, USA, for providing the antibody against LRPPRC. Furthermore, they would like to thank Dr. N. Kock for his scientific advice regarding the TAP technology. Funding sources included the Deutsche Forschungsgemeinschaft, Volkswagen Foundation, Thyssen Foundation, and Hermann and Lilly Schilling Foundation. A. Rakovic and A. Grünewald contributed equally to this study.

\section{References}

[1] J. Hardy, P. Lewis, T. Revesz, A. Lees, and C. Paisan-Ruiz, "The genetics of Parkinson's syndromes: a critical review," Current Opinion in Genetics and Development, vol. 19, no. 3, pp. 254$265,2009$.

[2] H. Shimura, M. G. Schlossmacher, N. Hattori et al., "Ubiquitination of a new form of $\alpha$-synuclein by parkin from human brain: implications for Parkinson's disease," Science, vol. 293, no. 5528, pp. 263-269, 2001.

[3] B. Tang, H. Xiong, P. Sun et al., "Association of PINK1 and DJ-1 confers digenic inheritance of early-onset Parkinson's disease," Human Molecular Genetics, vol. 15, no. 11, pp. 1816$1825,2006$.

[4] I. E. Clark, M. W. Dodson, C. Jiang et al., "Drosophila pink1 is required for mitochondrial function and interacts genetically with parkin," Nature, vol. 441, no. 7097, pp. 1162-1166, 2006.

[5] J. Park, S. B. Lee, S. Lee et al., "Mitochondrial dysfunction in Drosophila PINK1 mutants is complemented by parkin," Nature, vol. 441, no. 7097, pp. 1157-1161, 2006.

[6] Y. Yang, S. Gehrke, Y. Imai et al., "Mitochondrial pathology and muscle and dopaminergic neuron degeneration caused by inactivation of Drosophila Pink1 is rescued by Parkin," Proceedings of the National Academy of Sciences of the United States of America, vol. 103, no. 28, pp. 10793-10798, 2006.

[7] A. C. Poole, R. E. Thomas, S. Yu, E. S. Vincow, and L. Pallanck, "The mitochondrial fusion-promoting factor mitofusin is a substrate of the PINK1/parkin pathway," PLoS ONE, vol. 5, no. 4, Article ID e10054, 2010.

[8] E. Ziviani, R. N. Tao, and A. J. Whitworth, "Drosophila Parkin requires PINK1 for mitochondrial translocation and ubiquitinates Mitofusin," Proceedings of the National Academy of Sciences of the United States of America, vol. 107, no. 11, pp. 5018-5023, 2010.

[9] M. E. Gegg, J. M. Cooper, K.-Y. Chau, M. Rojo, A. H. V. Schapira, and J.-W. Taanman, "Mitofusin 1 and mitofusin 2 are ubiquitinated in a PINK1/parkin-dependent manner upon induction of mitophagy," Human Molecular Genetics, vol. 19, no. 24, pp. 4861-4870, 2010.

[10] C. Klein and M. G. Schlossmacher, "Parkinson disease, 10 years after its genetic revolution: multiple clues to a complex disorder," Neurology, vol. 69, no. 22, pp. 2093-2104, 2007.
[11] S. A. Miller, D. D. Dykes, and H. F. Polesky, "A simple salting out procedure for extracting DNA from human nucleated cells," Nucleic Acids Research, vol. 16, no. 3, p. 1215, 1988.

[12] K. Hedrich, J. Hagenah, A. Djarmati et al., "Clinical spectrum of homozygous and heterozygous PINK1 mutations in a large german family with parkinson disease: role of a single hit?" Archives of Neurology, vol. 63, no. 6, pp. 833-838, 2006.

[13] E. Moro, J. Volkmann, I. R. König et al., "Bilateral subthalamic stimulation in Parkin and PINK1 parkinsonism," Neurology, vol. 70, no. 14, pp. 1186-1191, 2008.

[14] M. Sena-Esteves, J. C. Tebbets, S. Steffens, T. Crombleholme, and A. W. Flake, "Optimized large-scale production of high titer lentivirus vector pseudotypes," Journal of Virological Methods, vol. 122, no. 2, pp. 131-139, 2004.

[15] E. Mortz, T. N. Krogh, H. Vorum, and A. Görg, "Improved silver staining protocols for high sensitivity protein identification using matrix-assisted laser desorption/ionization-time of flight analysis," Proteomics, vol. 1, no. 11, pp. 1359-1363, 2001.

[16] I. Topisirovic, N. Siddiqui, V. L. Lapointe et al., "Molecular dissection of the eukaryotic initiation factor $4 \mathrm{E}$ (eIF4E) export-competent RNP," EMBO Journal, vol. 28, no. 8, pp. 1087-1098, 2009.

[17] A. Rakovic, A. Grünewald, P. Seibler et al., "Effect of endogenous mutant and wild-type PINK1 on Parkin in fibroblasts from Parkinson disease patients," Human Molecular Genetics, vol. 19, no. 16, pp. 3124-3137, 2010.

[18] S. Mili and S. Piñol-Roma, "LRP130, a pentatricopeptide motif protein with a noncanonical RNA-binding domain, Is bound in vivo to mitochondrial and nuclear RNAs," Molecular and Cellular Biology, vol. 23, no. 14, pp. 4972-4982, 2003.

[19] A. Weihofen, B. Ostaszewski, Y. Minami, and D. J. Selkoe, "Pink1 Parkinson mutations, the Cdc37/Hsp90 chaperones and Parkin all influence the maturation or subcellular distribution of Pink1," Human Molecular Genetics, vol. 17, no. 4, pp. 602-616, 2008.

[20] E. M. Valente, S. Michiorri, G. Arena, and V. Gelmetti, "PINK1: one protein, multiple neuroprotective functions," Future Neurology, vol. 4, no. 5, pp. 575-590, 2009.

[21] A. J. Caplan, A. K. Mandal, and M. A. Theodoraki, "Molecular chaperones and protein kinase quality control," Trends in Cell Biology, vol. 17, no. 2, pp. 87-92, 2007.

[22] D. P. Narendra, S. M. Jin, A. Tanaka et al., "PINK1 is selectively stabilized on impaired mitochondria to activate Parkin," PLoS Biology, vol. 8, no. 1, Article ID e1000298, 2010.

[23] L. A. Mizzen, C. Chang, J. I. Garrels, and W. J. Welch, "Identification, characterization, and purification of two mammalian stres proteins present in mitochondria, grp 75, a member of the hsp 70 family and hsp 58, a homolog of the bacterial groEL protein," Journal of Biological Chemistry, vol. 264, no. 34, pp. 20664-20675, 1989.

[24] J. N. Dahlseid, R. Lill, J. M. Green, X. Xu, Y. Qiu, and S. K. Pierce, "PBP74, a new member of the mammalian 70-kDa heat shock protein family, is a mitochondrial protein," Molecular Biology of the Cell, vol. 5, no. 11, pp. 1265-1275, 1994.

[25] G. Szabadkai, K. Bianchi, P. Várnai et al., "Chaperonemediated coupling of endoplasmic reticulum and mitochondrial Ca channels," Journal of Cell Biology, vol. 175, no. 6, pp. 901-911, 2006.

[26] Q. Ran, R. Wadhwa, R. Kawai et al., "Extramitochondrial localization of mortalin/mthsp70/PBP74/GRP75," Biochemical and Biophysical Research Communications, vol. 275, no. 1, pp. 174-179, 2000.

[27] E. J. Davison, K. Pennington, C. C. Hung et al., "Proteomic analysis of increased Parkin expression and its interactants 
provides evidence for a role in modulation of mitochondrial function," Proteomics, vol. 9, no. 18, pp. 4284-4297, 2009.

[28] E. A. Craig, J. Kramer, and J. Kosic-Smithers, "SSC1, a member of the $70-\mathrm{kDa}$ heat shock protein multigene family of Saccharomyces cerevisiae, is essential for growth," Proceedings of the National Academy of Sciences of the United States of America, vol. 84, no. 12, pp. 4156-4160, 1987.

[29] W. Voos and K. Röttgers, "Molecular chaperones as essential mediators of mitochondrial biogenesis," Biochimica et Biophysica Acta, vol. 1592, no. 1, pp. 51-62, 2002.

[30] P. D'Silva, Q. Liu, W. Walter, and E. A. Craig, "Regulated interactions of mtHsp70 with Tim 44 at the translocon in the mitochondrial inner membrane," Nature Structural and Molecular Biology, vol. 11, no. 11, pp. 1084-1091, 2004.

[31] H. M. Li, T. Niki, T. Taira, S. M. M. Iguchi-Ariga, and H. Ariga, "Association of DJ-1 with chaperones and enhanced association and colocalization with mitochondrial Hsp70 by oxidative stress," Free Radical Research, vol. 39, no. 10, pp. 1091-1099, 2005.

[32] J. Jin, G. J. Li, J. Davis et al., "Identification of novel proteins associated with both $\alpha$-synuclein and DJ-1," Molecular and Cellular Proteomics, vol. 6, no. 5, pp. 845-859, 2007.

[33] C. C. Deocaris, S. Takano, D. Priyandoko et al., "Glycerol stimulates innate chaperoning, proteasomal and stressresistance functions: implications for geronto-manipulation," Biogerontology, vol. 9, no. 4, pp. 269-282, 2008.

[34] B. S. Park, Y. S. Song, S. B. Yee et al., "Phospho-ser 15-p53 translocates into mitochondria and interacts with $\mathrm{Bcl}-2$ and Bcl-xL in eugenol-induced apoptosis," Apoptosis, vol. 10, no. 1, pp. 193-200, 2005.

[35] L. De Mena, E. Coto, E. Sánchez-Ferrero et al., "Mutational screening of the mortalin gene (HSPA9) in Parkinson's disease," Journal of Neural Transmission, vol. 116, no. 10, pp. 1289-1293, 2009.

[36] J. Jin, C. Hulette, Y. Wang et al., "Proteomic identification of a stress protein, mortalin/mthsp70/GRP75: relevance to Parkinson disease," Molecular and Cellular Proteomics, vol. 5, no. 7, pp. 1193-1204, 2006.

[37] H. Koll, B. Guiard, J. Rassow et al., "Antifolding activity of hsp60 couples protein import into the mitochondrial matrix with export to the intermembrane space," Cell, vol. 68 , no. 6 , pp. 1163-1175, 1992.

[38] M. Y. Cheng, F. U. Hartl, J. Martin et al., "Mitochondrial heat-shock protein hsp60 is essential for assembly of proteins imported into yeast mitochondria," Nature, vol. 337, no. 6208, pp. 620-625, 1989.

[39] V. Veereshwarayya, P. Kumar, K. M. Rosen, R. Mestril, and H. W. Querfurth, "Differential effects of mitochondrial heat shock protein 60 and related molecular chaperones to prevent intracellular $\beta$-amyloid-induced inhibition of complex IV and limit apoptosis," Journal of Biological Chemistry, vol. 281, no. 40, pp. 29468-29478, 2006.

[40] R. Benecke, P. Strumper, and H. Weiss, "Electron transfer complexes I and IV of platelets are abnormal in Parkinson's disease but normal in Parkinson-plus syndromes," Brain, vol. 116, no. 6, pp. 1451-1463, 1993.

[41] A. H. V. Schapira, "Evidence for mitochondrial dysfunction in Parkinson's disease-a critical appraisal," Movement Disorders, vol. 9, no. 2, pp. 125-138, 1994.

[42] V. K. Mootha, P. Lepage, K. Miller et al., "Identification of a gene causing human cytochrome $\mathrm{c}$ oxidase deficiency by integrative genomics," Proceedings of the National Academy of Sciences of the United States of America, vol. 100, no. 2, pp. 605$610,2003$.
[43] F. Xu, C. Morin, G. Mitchell, C. Ackerley, and B. H. Robinson, "The role of the LRPPRC (leucine-rich pentatricopeptide repeal cassette) gene in cytochrome oxidase assembly: mutation causes lowered levels of COX (cytochrome c oxidase) I and COX III mRNA," Biochemical Journal, vol. 382, part 1, pp. 331-336, 2004.

[44] F. Sasarman, C. Brunel-Guitton, H. Antonicka et al., "LRPPRC and SLIRP interact in a ribonucleoprotein complex that regulates posttranscriptional gene expression in mitochondria," Molecular Biology of the Cell, vol. 21, no. 8, pp. 1315-1323, 2010.

[45] N. Sondheimer, J.-K. Fang, E. Polyak, M. J. Falk, and N. G. Avadhani, "Leucine-rich pentatricopeptide-repeat containing protein regulates mitochondrial transcription," Biochemistry, vol. 49, no. 35, pp. 7467-7473, 2010.

[46] M. P. Cooper, L. Qu, L. M. Rohas et al., "Defects in energy homeostasis in Leigh syndrome French Canadian variant through PGC-1 $\alpha /$ LRP130 complex," Genes and Development, vol. 20, no. 21, pp. 2996-3009, 2006.

[47] M. Ling, F. Merante, H. S. Chen, C. Duff, A. M. V. Duncan, and B. H. Robinson, "The human mitochondrial elongation factor tu (EF-Tu) gene: CDNA sequence, genomic localization, genomic structure, and identification of a pseudogene," Gene, vol. 197, no. 1-2, pp. 325-336, 1997.

[48] S. M. Chuang, LI. Chen, D. Lambertson, M. Anand, T. G. Kinzy, and K. Madura, "Proteasome-mediated degradation of cotranslationally damaged proteins involves translation elongation factor 1A," Molecular and Cellular Biology, vol. 25, no. 1, pp. 403-413, 2005.

[49] N. Shiina, Y. Gotoh, N. Kubomura, A. Iwamatsu, and E. Nishida, "Microtubule severing by elongation factor $1 \alpha$," Science, vol. 266, no. 5183, pp. 282-285, 1994.

[50] S. R. Gross and T. G. Kinzy, "Translation elongation factor $1 \mathrm{~A}$ is essential for regulation of the actin cytoskeleton and cell morphology," Nature Structural and Molecular Biology, vol. 12, no. 9, pp. 772-778, 2005.

[51] T. Tong, J. Ji, S. Jin et al., "Gadd45a expression induces bim dissociation from the cytoskeleton and translocation to mitochondria," Molecular and Cellular Biology, vol. 25, no. 11, pp. 4488-4500, 2005.

[52] L. Valente, V. Tiranti, R. M. Marsano et al., "Infantile encephalopathy and defective mitochondrial DNA translation in patients with mutations of mitochondrial elongation factors EFG1 and EFTu," American Journal of Human Genetics, vol. 80, no. 1, pp. 44-58, 2007.

[53] F. Gillardon, "Interaction of elongation factor 1-alpha with leucine-rich repeat kinase 2 impairs kinase activity and microtubule bundling in vitro," Neuroscience, vol. 163, no. 2, pp. 533-539, 2009. 


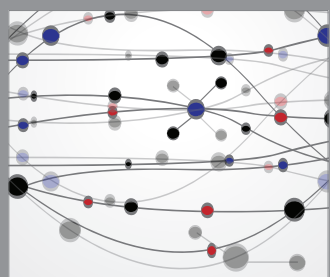

The Scientific World Journal
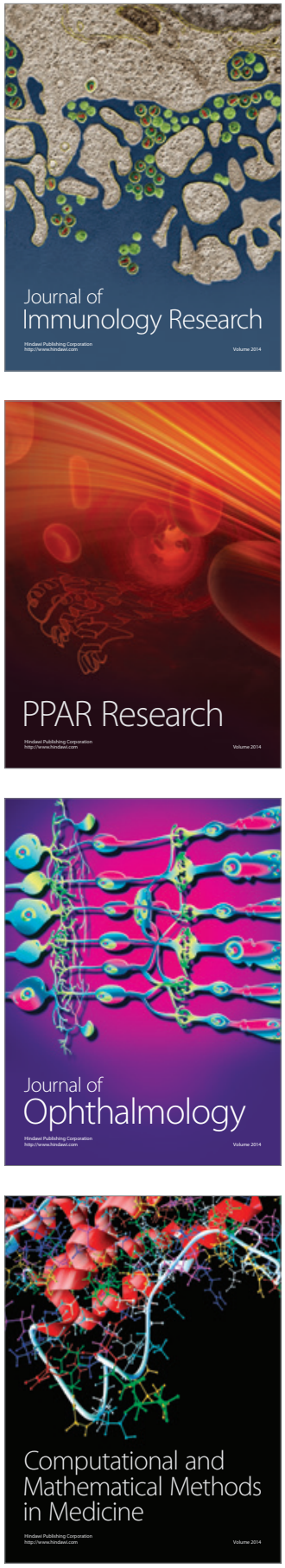

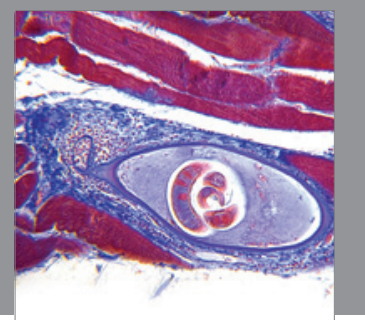

Gastroenterology

Research and Practice
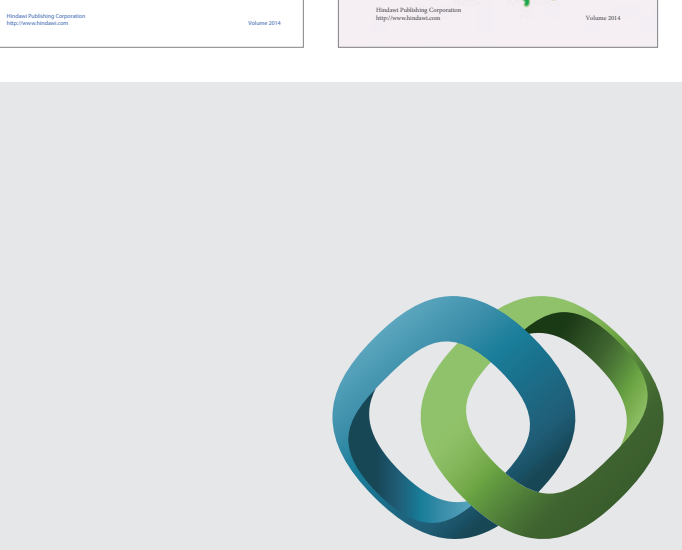

\section{Hindawi}

Submit your manuscripts at

http://www.hindawi.com
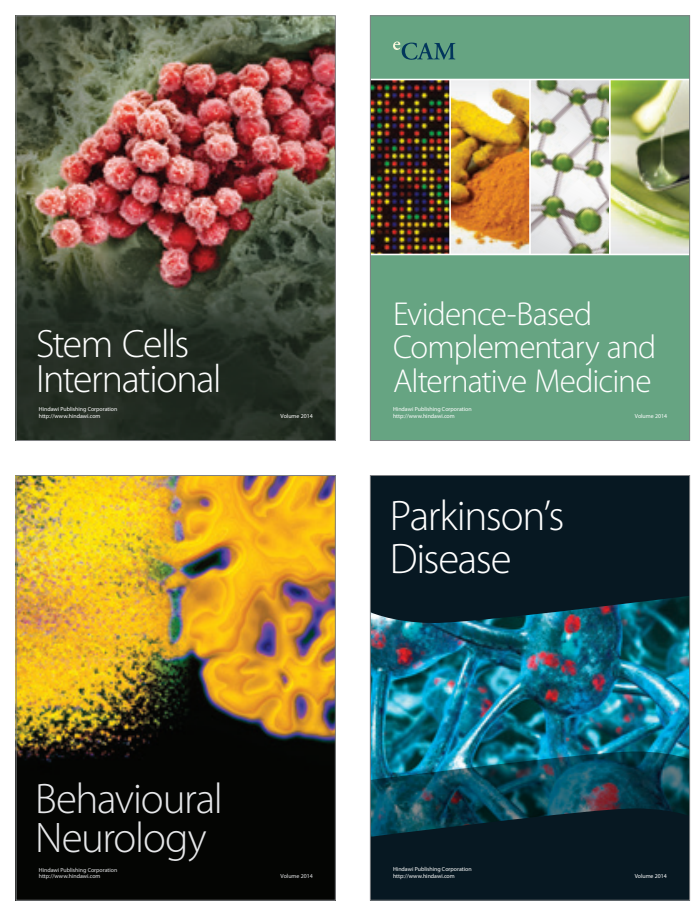

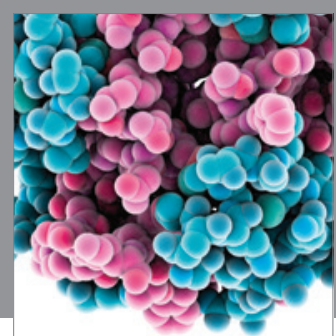

Journal of
Diabetes Research

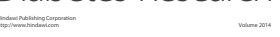

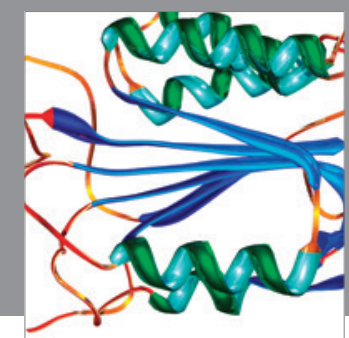

Disease Markers
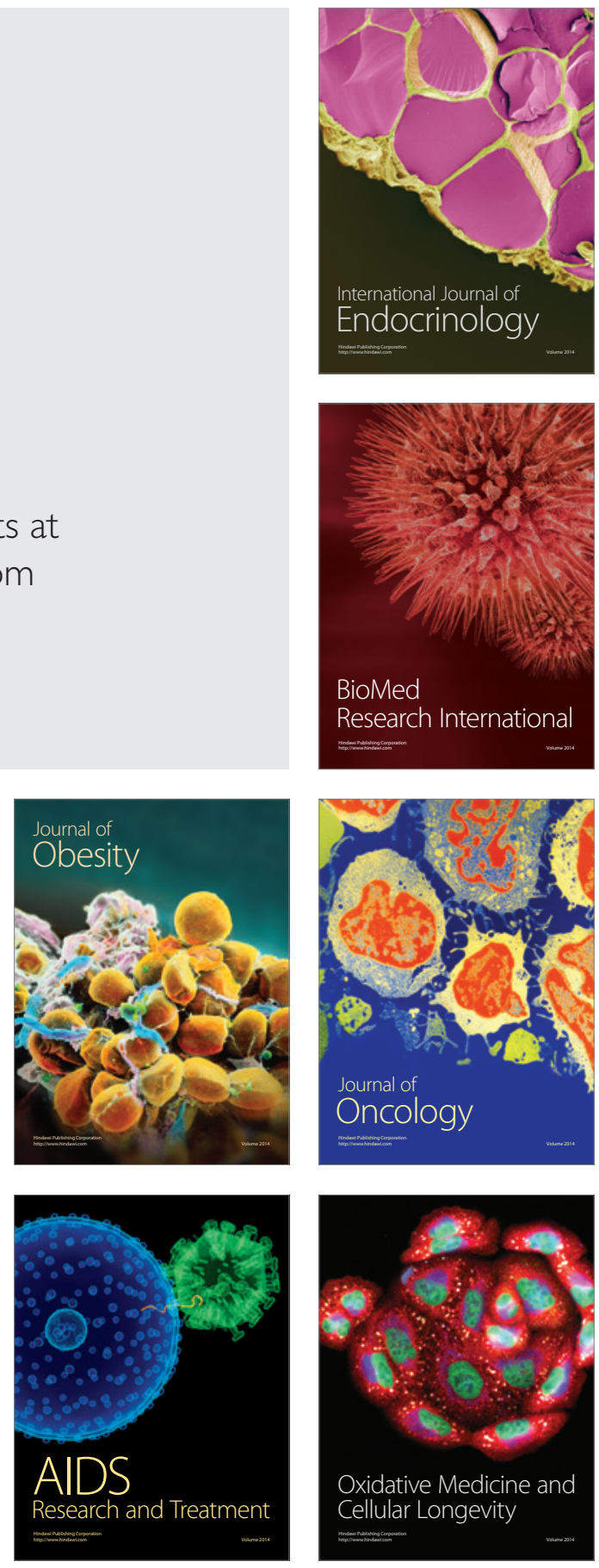